\title{
Recuperando la función endotelial
}

\author{
Recovering endothelial function
}

\author{
Alfonso Bryce Moncloa ${ }^{1}$, Enrique C. Morales-Villegas², Juan Urquiaga Calderón ${ }^{3}$, \\ Cesar Larrauri-Vigna ${ }^{4}$ \\ 'Médico Cardiólogo, Académico Asociado de la Academia Nacional de Medicina, Presidente del Colegio Panamericano del Endotelio, \\ Miembro del Comité Institucional de Investigaciones del Comité Ejecutivo de la Sociedad Latino Americana de Hipertensión Arterial y del \\ Colegio Americano de Cardiología (ACC). Correo-e: cardiogolf1@yahoo.es. \\ ${ }^{2}$ Médico Internista y Cardiólogo, Fundador y Director del Centro de Investigación Cardiometabólica de Aguascalientes. Correo-e: drmorvi@ \\ prodigy.net.mx \\ ${ }^{3}$ Médico Cardiólogo, Maestro en Salud Pública, Miembro de la Sociedad Peruana de Hipertensión Arterial, Colegio Panamericano del \\ Endotelio, Colegio Americano de Cardiologia (ACC). Correo-e: jurquiaga@hh-peru.com. \\ ${ }^{4}$ Médico Cardiólogo, Miembro de la Sociedad Peruana de Cardiología, Sociedad Peruana de Hipertensión, Sociedad Peruana de Medicina \\ Interna, Colegio Panamericano del Endotelio. Correo-e: cesarlarrauri@hotmail.com.
}

\begin{abstract}
Resumen
La enfermedad ateroesclerótica comienza a temprana edad. La presencia de factores de riesgo, como la hipertensión arterial, tabaquismo, dislipidemia y diabetes mellitus, a lo que hoy se suman la obesidad y el sindrome metabólico, originan desde el inicio disfunción endotelial, incremento del estrés oxidativo e inflamación, con aparición temprana de células espumosas, estrías grasas y placas de ateroma. Placas que en algunos de los casos son consideradas vulnerables, con un riesgo de sufrir erosión y rotura, el fenómeno aterotrombótico y un evento vascular agudo, como el síndrome coronario agudo y el accidente cerebrovascular. Al manejar y tratar los factores de riesgo cardiovasculares, síndrome metabólico, marcadores precoces del riesgo cardiovascular, se podría enlentecer o retardar el envejecimiento (injuria endotelial) de los órganos blanco, probablemente sin reducción de la mortalidad, pero logrando tres enfoques fundamentales con la detección temprana de la aterotrombosis subclínica: prevenir eventos cardiovasculares, brindar protección de los órganos blanco y mejorar la calidad de vida.
\end{abstract}

Palabras clave: Disfunción endotelial, ateroesclerosis coronaria, enfermedad coronaria.

\section{Abstract}

Atherosclerosis starts early in life. The presence of risk factors like hypertension, smoking, dyslipidemia and diabetes as well as obesity and metabolic syndrome accelerates its progress. These factors generate endothelial dysfunction, oxidative stress and inflammation, with early appearance of foamy cells, fatty streaks and atheromatous plaques. These plaques are vulnerable to erosion and rupture, the so called atherothrombotic phenomenon, leading to acute vascular events like acute coronary or cerebrovascular syndromes. Managing and treating the metabolic syndrome, early markers of cardiovascular risk, could slow down end organ aging and damage, probably without reduction of mortality but focusing on three fundamental aspects of early detection of subclinical atherothrombosis: prevention of cardiovascular events, protection of end organs, and improvement of quality of life.

Keywords: Endothelial dysfunction, coronary atherosclerosis, coronary artery disease.

An Fac med. 2014;75(4):367-73 / doi: http://dx.doi.org/10.15381/anales.v75i4.10859

La valoración del riesgo cardiovascular se inicia con los estudios de Framingham, hace más de 50 años ${ }^{(1)}$. A través del tiempo, el cuidadoso monitoreo de los participantes del Estudio de Framingham ha llevado a la identificación de los principales factores de riesgo para enfermedades cardiovasculares (presión arterial alta, niveles altos de colesterol, fumar, obesidad, diabetes e inactividad física) ${ }^{(2)}$. Aunque el grupo original de participantes fue en su mayoría caucásico, la importancia de los principales factores de riesgo de enfermedad cardiovascular identificados en este grupo, ha demostrado ser algo que aplica casi de manera universal en otros grupos raciales. Sin embargo, actualmente tenemos otros parámetros de evaluación que debemos considerar: componentes del síndrome metabólico, factores genéticos, nuevos biomarcadores y métodos de detección por imágenes. Todos ellos aislados o conjugados en los llamados puntaje de riesgo, ayudarán a una mejor estratificación de los pacientes, principalmente en la detección de aquellos grupos de pacientes asintomáticos considerados de riesgo intermedio. Por consiguiente, se debe- ría considerar no solamente un control agresivo de la presión arterial (PA) y de los otros factores de riesgo cardiovascular, sino también qué drogas son la mejor selección para prevenir eventos cardiovasculares $(\mathrm{CV})$, brindar protección de los órganos blanco y mejorar la calidad de vida.

Existe escasa evidencia acerca de la utilización de los diferentes métodos de evaluación (puntajes) de riesgo en la población latinoamericana. Ruiz y colaboradores ${ }^{(3)}$, aplicando el tradicional puntaje de Framingham a la población 
peruana, han determinado que alrededor de $60 \%$ de los sujetos evaluados se encuentran catalogados como de riesgo cardiovascular bajo, predominando en esta categoría el sexo femenino $(81,2 \%$ en mujeres versus $41,5 \%$ en varones). En contraste, la población de riesgo alto bordea el $20 \%$, cifra alta para nuestra realidad sanitaria. Pero, lo más resaltante es que en el lapso de siete años (2004 al 2011) se ha incrementado en casi 3\%. La edad calculada para el sexo masculino, era de 55,9 años, mientras que la edad biológica promedio que le debía corresponder a dicha población era de 49,8 años, en el año 2004, existiendo un envejecimiento mayor en 6,1 años; en el caso de la mujer, la diferencia fue de 3,8 años mayor que su edad biológica. Seis años después (2010), la población masculina presentaba una diferencia de 6,2 años mayor a su edad biológica promedio, y en la mujer esta diferencia era de 4 años mayor. Este envejecimiento de las arterias en la población peruana es producto de los estilos de vida desfavorables de la población desprotegida, fundamentalmente por desconocimiento y falta de educación sobre los riesgos que encierran los llamados estilos de vida del progreso económico.

Hoy en día, la estrategia es identificar al paciente vulnerable, entendido como el individuo susceptible al desarrollo de ateroesclerosis y por lo tanto con mayor riesgo de sufrir sus complicaciones. La prevención primaria efectiva requiere una estimación adecuada del riesgo para seleccionar el tratamiento más apropiado. Resulta atractiva entonces una estrategia basada en la detección temprana de la aterosclerosis subclínica, complementando a la aproximación tradicional del riesgo mediante los factores cardiovasculares clásicos. Por ejemplo, el grupo de expertos que conforman The Screening for Attack Prevention and Education Task Force (Grupo SHAPE) (4) postula incluir en el proceso de estratificación, como herramientas diagnósticas, estudios como la medición por ultrasonido del espesor medio-intimal carotídeo, la cuantificación del puntaje de calcio por tomografía computada multicorte y el perfil de riesgo otorgado a través de la medición de marcadores séricos, principalmente PCRus.

Las causas de alteración de la función endotelial son múltiples; lo importante es restaurar la función endotelial en forma global con el manejo integral de los factores de riesgo. Más de 50\% de las muertes coronarias no presentan síntomas previos; la muerte es la primera manifestación, en mujeres en $64 \%$. El $50 \%$ de los ataques cardíacos no ocurren en personas con riesgo cardiovascular elevado. La disfunción endotelial es la gran responsable ${ }^{(5,6)}$.

Diferentes fármacos tienen efectos probables sobre la producción de óxido nítrico, por citar, inhibidores de la enzima convertidora de angiotensina II (IECAs), bloqueadores del receptor AT1 de la angiotensina II (BRATs), bloqueadores de los canales de calcio (BCC), estatinas, beta bloqueadores (BB) con efecto vasodilatador ${ }^{(7)}$.

El estrés oxidativo es una desregulación molecular en el metabolismo de las especies reactivas de oxígeno (ROS), teniendo un rol principal en la patogénesis de la ateroesclerosis, inflamación vascular y disfunción endotelial. Así, las nicotinamide adenine dinucleotide phosphate (NADPH) (o dinucléotido de nicotinamida y adenina) oxidasas son una importante meta terapéutica. Interfieren e inhiben, con la activación y expresión de las NADPH oxidasas, los bloqueadores del sistema renina angiotensina aldosterona (SRAA) (principalmente los IECAs/BRATs), a los que nos vamos a referir. Por lo tanto, nuestros esfuerzos deben ser enfocados sobre los inhibidores de la NADPH oxidasas que generan moléculas de pequeño peso molecular, permitiendo una inhibición selectiva de los homólogos de la NADPH oxidasas; así, el tratamiento es más razonable y potencialmente más efectivo que los no selectivos.

Debido a la complejidad de los mecanismos comprometidos en la activación de las NADPH oxidasas, estas enzimas pueden ser controladas en diferentes niveles de su actividad, a saber, disminuyendo su expresión, bloqueando la translocación, previniendo su fosforilación, señal de transducción, finalmente, tratar solo de inhibir las isoformas específicas. En forma práctica, varias drogas, como las que hemos mencionado, logran estos objetivos, disminuyendo la función de las NADPH oxidasas.

La disfunción endotelial es causada por una sobreproducción de ROS, conduciendo a una disminución de las biodisponibilidad del óxido nítrico. Numerosos estudios han mostrado mejora de la disfunción endotelial y estrés oxidativo usando diferentes estrategias de tratamiento, las cuales incluyen estatinas, IECAs, BRATs, BCC, BB de última generación, inhibidores de PD5 y otros. Son también importantes la modificación del estilo de vida, mejora en la alimentación, la actividad física, dejar fumar, entre otros. Las terapias que logren mejorar la disponibilidad del óxido nítrico $(\mathrm{NO})$ para revertir la disfunción endotelial pueden conducir a mejorar el manejo de enfermedades como la hipertensión y diabetes.

Debemos considerar de que estamos en la era cardiometabólica. Por consiguiente, no olvidar que el riesgo es global y debe ser enfocado bajo estos conceptos, a saber, control de los factores de riesgo tradicionales, nuevas perspectivas, nuevas metas en el manejo y terapia de los factores de riesgo cardiovascular, nuevas guías integradas para la reducción del riesgo cardiovascular total (riesgo integrado) ${ }^{(8)}$.

\section{OBESIDAD, SÍNDROME \\ METABÓLICO Y RIESGO CARDIOVASCULAR}

El síndrome metabólico no es una enfermedad nueva. Su descripción tuvo lugar hace al menos 80 años (en la década de los años veinte) por parte de Kylin ${ }^{(9)}$, un médico sueco que definió la asociación entre hipertensión, hiperglucemia y gota. 
El síndrome metabólico se está convirtiendo en uno de los principales problemas de salud pública del siglo XXI, asociado a un incremento de 5 veces en la prevalencia de diabetes tipo 2 , y de 2 a 3 veces en la de enfermedad cardiovascular (ECV).

La Asociación Americana del Corazón / Instituto Nacional del Corazón, pulmón y sangre (AHA/NHLBI) ha publicado declaraciones científicas acerca del síndrome metabólico, en las que se recoge una clasificación ATP-III ${ }^{(10)}$ actualizada. No se considera necesario el criterio del incremento del perímetro de la cintura en los pacientes en quienes hay otros tres factores de riesgo. La definición ATP-III también contempla una disminución del valor umbral del perímetro de la cintura en lo relativo al riesgo, sobre todo en las personas de origen asiático-americano. Esta versión actualizada de la definición ATP-III y los nuevos criterios propuestos por la International Diabetes Federation (IDF) permiten establecer el diagnóstico de síndrome metabólico básicamente en los mismos pacientes.

Nuestra población es multiétnica y por lo tanto la cintura tiene una amplia variación en las diferentes regiones (costa, sierra, selva), en la población migrante y en la tercera edad. No existen estudios poblacionales en todas estas regiones y grupos poblacionales y tomar como parámetro principal a la cintura para definición del síndrome metabólico resulta dejar de lado otros factores, como la elevación de triglicéridos o HDL bajo, que son frecuentes en nuestra población de altura. Por lo tanto, creemos por conveniente plantear como definición de síndrome metabólico para nuestra población la definición de Scientific Statement. Diagnosis and Management of the Metabolic Syndrome. American Heart Association/ National Heart, Lung, and Blood Institute (AHA/NHLBI) (ATP modificado) ${ }^{(11)}$.

Prediabetes significa una elevación de la glucosa plasmática por encima de la glicemia basal, pero debajo de los niveles del diagnóstico de la diabetes clínica, o favorecer la intolerancia a la glucosa. Ambos son condicionantes de diabetes mellitus tipo 2 (DM) y el riesgo es aún mayor cuando ambas están juntas. La prediabetes comúnmente se asocia al síndrome metabólico (SM); ambos casos son muy cercanos a la obesidad. Los mecanismos por los cuales la obesidad predispone a prediabetes y SM no son bien comprendidos, pero frecuentemente tienen el mismo fondo metabólico. La resistencia a la insulina es un factor, la inflamación sistémica engendrada por la obesidad puede ser otra. La prediabetes tiene un menor impacto sobre la enfermedad microvascular; las drogas para disminuir la glicemia pueden dilatar la conversión a diabetes, pero su uso a largo tiempo puede ayudar a disminuir el compromiso macrovascular. No se ha evaluado el efecto de las drogas empezadas tempranamente en la prediabetes. La prediabetes, de alguna manera, puede ser predictora de enfermedades macrovasculares, pero la mayoría de estas asociaciones parecen ser mediadas a través del SM ${ }^{(12)}$.

El alcance clínico preferido para prevenir la enfermedad cardiovascular es tratar todos los factores de riesgo metabólicos. Es importante modificar los estilos de vida, especialmente reducir de peso y mejorar la actividad física. Cuando se contempla la terapia en caso de que el SM está presente, la primera consideración es la prevención cardiovascular.

La prevalencia de DM se está incrementando progresivamente en todo el mundo. Las mayores complicaciones en DM son cardiovasculares, tanto microvasculares como macrovasculares.

El nivel sanguíneo de glucosa elevado es uno de los componentes del SM; otros son la obesidad abdominal (cintura abdominal, caracterizando la obesidad visceral), presión arterial elevada, triglicéridos elevados y reducción del HDL colesterol. De esto 5 componentes, 3 confieren el diagnóstico de SM. La mayoría de los individuos con SM tiene obesidad abdominal. El tejido adiposo abundante libera un exceso de ácidos grasos libres y una variedad de adipoquinas (la única buena es la adiponectina), que aumentan el riesgo metabólico y predispone tanto a la DM como ECV (13).

La mayoría de las personas con ambas condiciones son obesas. Un incremento del tejido adiposo resulta en elevación de los ácidos grasos libres (FFA) circulantes y otras adipoquinas tóxicas. En ambos casos se produce un estado proinflamatorio y protrombótico. Un incremento de los FFA induce a la resistencia a la insulina (IR) en los músculos, lo cual contribuye a la elevación de la glucosa plasmática. A largo plazo, los niveles altos de FFA circulantes pueden dañar la función de las células beta por lipotoxicidad; esto también provocará un incremento significativo de glucosa. Los FFA elevados probablemente contribuyan a incrementar la salida de glucosa del hígado, empeorando la hiperglicemia. Así también aumentarían los triglicéridos, lo cual evitaría el aumento del HDL colesterol. La obesidad incrementa los niveles de presión arterial, aunque los mecanismos para este efecto no han sido bien dilucidados. Un estado proinflamatorio frecuentemente predispone a la prediabetes por intensificación de la resistencia a la insulina. Muchos investigadores también creen que un estado proinflamatorio predispone a la enfermedad cardiovascular, así como a un estado protrombótico. La obesidad predispone tanto a la prediabetes como al SM.

\section{CONTROL DE LA HIPERTENSIÓN ARTERIAL}

Son numerosas las publicaciones que definen a la ateroesclerosis como una enfermedad vascular de origen inflamatorio, que puede presentarse de forma concomitante con otras patologías como la hipertensión arterial.

La angiotensina II ha demostrado durante los últimos años, además de poseer un potente efecto sobre la presión arterial, ser una importante citoquina proinflamatoria a nivel vascular, 
capaz de inducir disfunción endotelial y condicionando estrés oxidativo y cambios estructurales y funcionales de la pared vascular.

El rol significativo de los IECAs y BRATs en la patogénesis de la ateroesclerosis y sus efectos clínicos beneficiosos han sido demostrados en muchas investigaciones. Los IECAs mejoran la función del endotelio por varios mecanismos, incluyendo las propiedades antioxidantes, efectos beneficiosos sobre la fibrinólisis, disminución de la actividad de la angiotensina II, incremento de la bradiquinina, disminución de los productos de glicación avanzados (AGE) en los diabéticos, entre otros. Los BRATs acompañan en estas propiedades de los IECAs, dando una protección especial al tener mayor actividad de los receptores AT2 y AT1-7. Los BCC también tienen propiedades favorables en la recuperación de la función endotelial. Los BB de cuarta generación, principalmente los que liberan producción de óxido nítrico tienen estas propiedades favorables para el control del estrés oxidativo e inflamación vascular ${ }^{(14)}$.

Un análisis crítico a las nuevas Guías Americanas (JNC8) y que deberíamos tener en cuenta en el manejo de la hipertensión arterial es que, al igual que las guías europeas, antes de empezar el tratamiento farmacológico de la hipertensión arterial, la primera indicación debe ser la modificación del estilo de vida, a través de medidas ampliamente reconocidas: dejar de fumar, ejercicio físico regular aeróbico, reducción del peso, reducción de la ingesta de grasas saturadas y totales, de la ingesta de sal y de la ingesta excesiva de alcohol, e incremento de la ingesta de frutas y vegetales. Es fundamental la estratificación del riesgo del paciente, con base principalmente en las Guías Latinoamericanas, de acuerdo al II Consenso Latinoamericano de Hipertensión Arterial en el 2008 y publicadas en el 2009. Estas guías están basadas y son muy parecidas a las europeas, donde observamos que de acuerdo a los niveles de presión arterial, a veces sin llegar al estadio I sino en el estadio considerado como prehipertensión o presión arterial normal alta, el riesgo aumenta según los factores de riesgo existentes, principalmente en pacientes con tres factores de riesgo, daño subclínico de órgano blanco o cuadro compatible con síndrome metabólico y, sin ninguna duda, en los diabéticos. Este grupo de personas demuestran ya cierto grado de deterioro cardiovascular, en quienes debería considerarse el tratamiento farmacológico de inicio asociado a los cambios en el estilo de vida.

Un concepto interesante considerado en las actuales Guías Latinoamericanas ${ }^{(15)}$, como aporte principal, es el llamado 'condiciones sociales' en riesgo, según el cual, el hábitat, las condiciones de cultura y la facilidad del servicio médico podrían variar el riesgo del paciente. Por tal razón, estas guías mencionan que, entre los factores de riesgo tradicionales, las condiciones socioeconómicas en América Latina deben tener una particular atención.

Las nuevas Guías, tanto las Europeas del $2013^{(16)}$ y un grupo del Panel de Miembros del JNC $8^{(17)}$ han considerado, para poblaciones especiales con riesgo alto como los diabéticos, cifras de $140 / 85 \mathrm{mmHg}$ y $140 / 90 \mathrm{mmHg}$, respectivamente, lo que podría considerarse como un retroceso, ya que estaríamos dejando a un grupo de pacientes de probable riesgo alto desprotegidos ${ }^{(18)}$. Los marcadores precoces de la enfermedad están presentes mucho antes de la elevación sostenida de la presión; por lo tanto, la hipertensión no puede ser clasificada por elevación en los umbrales normales de la presión arterial en forma aislada ${ }^{(19)}$.

Recientemente, en un nuevo metaanálisis que incluye más de 700000 pacientes, publicado en la revista Neurology (abril 2014) ${ }^{(20)}$, la hipertensión limítrofe, conocida como prehipertensión, fue asociada con un incremento significativo en el riesgo de accidente cerebrovascular (ACV). En este sentido, la primera intervención ya mencionada y reiterada desde años previos es la modificación del estilo de vida (21). Esto muestra la evidencia de que los médicos y pacientes deben tomar seriamente la presión arterial limítrofe, sobretodo en pacientes de riesgo de ACV por historia familiar, diabetes, u otras condiciones vasculares.

Entre estas complejidades, probablemente lo más importante a considerar es el valor óptimo de la presión arterial para el tratamiento antihipertensivo, particularmente en pacientes de 'riesgo alto', en quienes un pequeño descenso de la presión arterial puede significativamente incrementar el beneficio o riesgo.

Otro punto controversial, es el temor de la exacerbación de la curva J ${ }^{(22)}$. La llamada curva J es la mala perfusión coronaria durante la diástole y es más evidente cuanto mayor es la presión de pulso, lo cual aumenta el riesgo coronario. Posiblemente se debió analizar mejor el tema, tipificando al paciente de riesgo o llamado también frágil; no todo paciente diabético con enfermedad coronaria se comporta igual. Más importante que conocer si la curva J existe o no, es identificar al paciente frágil, y como pacientes frágiles se debe considerar a los pacientes coronarios no revascularizados, los que tienen hipertensión sistólica aislada pronunciada, los que presentan hipotensión ortostática, quienes tienen disfunción ventricular izquierda acentuada o aquellos con un tiempo mayor de evolución coronaria y diabetes ${ }^{(23)}$. Las nuevas guías han debido explicar mejor lo que significa un tratamiento temprano en los pacientes de riesgo alto, que sería a todas luces beneficioso, frente a un tratamiento tardío, de muchos años de enfermedad, con daño de órgano blanco significativo, donde el tratamiento agresivo no sería beneficioso. Esto conduciría a la intervención temprana en la historia natural de la hipertensión, antes de que se dañe el órgano blanco o cuando el daño subclínico está presente ${ }^{(24)}$. El viejo paradigma, mientras más bajo mejor, ha sido reemplazado por el nuevo paradigma, cuando más temprano... mejor ${ }^{(21)}$. 
En suma, reducir las cifras de presión arterial tempranamente, de una manera rápida y eficaz, comporta una disminución del riesgo vascular y mejora del pronóstico de los pacientes hipertensos.

Comentarios recientes sobre las Guías Americanas de HTA (JNC8) ${ }^{(25)}$ ameritan presentar algunas de sus opiniones, a saber, que a los sesenta años, menos de $150 \mathrm{mmHg}$ de presión arterial sistólica (PAS) sería de beneficio sin excesivo riesgo, basado en los estudios SHEP y Syst Eur al respecto. No se excluye la posibilidad de que menos de $140 \mathrm{mmHg}$ es mejor que menos de 150 $\mathrm{mmHg}$. Menos de $150 \mathrm{mmHg}$ es mejor que más de $150 \mathrm{mmHg}$ en el estudio SHEP, pero no es así en el estudio Syst Eur, donde las cifras más bajas fueron mejores. Entre los 60 y 80 años, cifras de $140 \mathrm{mmHg}$ pueden ser mejores que $150 \mathrm{mmHg}$, y en algunos casos menos de $140 \mathrm{mmHg}$ sería mejor. Otros expertos consideran que el JNC 8 defiende la cifra de $150 \mathrm{mmHg}$ para evitar el riesgo. Se concluiría que el juicio clínico es crítico, y si el médico considera que su paciente se beneficia con menos cifras, se le debe respetar. Es un mensaje a reforzar, hasta que se pueda probar convincentemente que lograr las metas más apropiadas es lo mejor.

Una reflexión final, la libertad del médico para el tratamiento de un paciente ha sido notablemente limitada por el concepto de la medicina basada en la evidencia. Claramente, todos desearían practicar de acuerdo a la mejor evidencia disponible. Pero, se ha aceptado que basado en la evidencia significa aquello que deriva de estudios clínicos aleatorizados, y preferentemente a doble ciego. En el mundo real de pacientes con múltiples enfermedades y que están recibiendo diferentes drogas, la práctica de la medicina basada en la evidencia es extremadamente difícil. Para cada paciente se debe efectuar un balance entre los riesgos y beneficios de cada tratamiento. La buena práctica todavía requiere la libertad clínica de los médicos ${ }^{(27)}$.
Para la prevención y tratamiento de la enfermedad hipertensiva y cardiovascular, debemos considerar que, al manejar y tratar los factores de riesgo cardiovasculares (FRCV), síndrome metabólico (SM), marcadores precoces del riesgo cardiovascular, daño de órgano blanco (DOB) subclínico, se podría enlentecer o retardar el envejecimiento (injuria endotelial) e instalación en los DOB, probablemente no la mortalidad, pero sí mejorar la calidad de vida.

\section{CONTROL DE LA DISLIPIDEMIA. ENFOQUE EN ESTATINAS}

Se han realizado numerosos estudios sobre las estatinas -inhibidores de la hidroximetilgliceril coenzima A (HMGCoA) - y se ha determinado su influencia benéfica sobre la prevención de la disfunción endotelial. Esto es debido a la disminución de los niveles de LDLcolesterol, lo cual incrementa la vasodilatación dependiente del óxido nítrico (NO) e inhibe el estrés oxidativo en la pared del vaso. Así mismo, los efectos pleiotrópicos de las estatinas tienen la capacidad de limitar directamente el estrés oxidativo sobre el vaso sanguíneo. Múltiples estudios indican el rol inhibitorio de la NAPDH oxidasa, como un mecanismo mayor del efecto pleiotrópico de las estatinas.

$\mathrm{Al}$ mismo tiempo, las estatinas también han mostrado una disminución de la expresión de numerosas subunidades y homólogos de la NAPDH oxidasas; por consiguiente, sus efectos son multifocales. Estos efectos son reflejados por observación en numerosos modelos de enfermedad vascular asociados a estrés oxidativo ${ }^{(14)}$. Las estatinas son las más ampliamente usadas para controlar la ECV por sus efectos pleiotrópicos, independientemente de disminuir los lípidos ${ }^{(27)}$.

Las estatinas muestran sus efectos antiinflamatorios sobre la pared vascular a través de una variedad de mecanismos moleculares de los sistemas inmunes innatos y adaptativos, su impacto sobre los niveles circulan- tes de citoquinas proinflamatorias y su efecto sobre las moléculas de adhesión, inhibiendo la vía del mevalonato y formación de isoprenoides. Las estatinas cuentan para el incremento de la biodisponibilidad del $\mathrm{NO}$ y el mejoramiento del estado redox vascular y miocárdico, por mecanismos múltiples y diferentes (directa o indirectamente a través de la disminución del colesterol LDL). Un sinnúmero de estudios aleatorizados han mostrado que las estatinas previenen los ECV, tanto en la prevención primaria como secundaria, no solo por la vía de sus efectos en reducir el colesterol LDL, sino también por su potencial antiinflamatorio, y el enfoque especial es sobre su impacto en los sistemas inmunológicos innatos y adaptativos.

Dado el hecho de que la ateroesclerosis es una enfermedad multivariable, con varias moléculas comprometidas en cada estadio, es muy difícil encontrar un tratamiento efectivo. Sin embargo, las estatinas han probado ser el tratamiento más efectivo, porque interfiere en la mayoría de los componentes críticos del proceso ateroesclerótico, probando tener efectos beneficiosos.

En un momento en el cual la nueva Guía ATP-IV aún no es asimilada por los médicos y sobre ello se ha iniciado la investigación y uso clínico de nuevas terapias biológicas, anti-PCSK9, antiapoB y anti-MTP, diseñadas para el tratamiento de la hipercolesterolemia y la enfermedad cardiovascular aterosclerosa, al menos, un concepto está muy claro, a saber, los inhibidores de la HMGCoA-R o estatinas son la piedra angular en el tratamiento farmacológico orientado a reducir el riesgo cardiovascular asociado a ateroesclerosis. Siendo las estatinas el grupo farmacológico que reduce con mayor intensidad la concentración de LDL en plasma, amén de otras acciones no lipídicas o pleiotrópicas, su eficacia terapéutica se explica si aceptamos que las LDL en concentración suprafisiológica, al modificarse por oxidación, se transforman en epítopes que provocan en conjunto con otros factores de lesión endotelial 
y vascular una secuencia fisiopatológica y anatomopatológica caracterizada por ateroesclerosis, activación, disfunción y lesión endoteliales seguida de aterosclerosis y aterotrombosis. Así, hasta hoy, las estatinas son nuestra arma farmacológica más importante para la reducción del riesgo cardiovascular asociado a aterosclerosis. En este artículo dedicado al rescate endotelial se revisa, inicialmente, las características farmacológicas de las estatinas, específicamente sus mecanismos de acción y los efectos que explican sus acciones lipídicas y no lipídicas o pleiotrópicas. Estos conceptos básicos se complementan con un breve resumen de las recomendaciones ATP-IV para la prescripción eficiente de estatinas. Como se analizará, estas recomendaciones encierran el estado del arte de 33 años de investigación clínica de este escenario terapéutico y es por eso se las presenta aquí.

\section{ESTATINAS. MECANISMOS DE ACCIÓN}

Las estatinas, al inhibir a la HMGCoA-R y con ello la síntesis de mevalonato, colesterol e isoprenoides, provocan un engaño celular en el cual los mecanismos que les permiten reducir la concentración de LDL en plasma son los siguientes: a) reducción de la síntesis hepática de colesterol y por ende reducción en la lipidación y síntesis de la lipoproteína VLDL, precursora de IDL y LDL; b) reducción en la concentración de colesterol en las membranas del hepatocito con desinhibición del SREBP y activación en la transcripción, síntesis y expresión del R-LDL con mayor captación de IDL en apo E y de LDL en apo B100; y, c) incremento en la captación hepática de IDL y LDL con mayor eliminación hepatobiliar de colesterol esterificado.

Así, desde la publicación de Hiroshi Mabushi, en 1981, en la cual demostró por primera vez en la clínica el impacto favorable de una estatina en los niveles de lípidos de individuos con hipercolesterolemia familiar, han transcurrido 33 años de una historia que se podría denominar el rescate endotelial de su principal patógeno, los niveles suprafisiológicos de LDL. Los años por venir nos preparan la segunda parte de esta historia cuya interrogante central es la siguiente. ¿Más allá de las estatinas, las terapias biológicas reductoras de LDL reducirán en paralelo la incidencia de enfermedades cardiovasculares ateroesclerosas? La predicción es que sí, en la medida en que podamos en forma precoz, sostenida y de largo plazo mantener el nivel de LDL cercano al valor biológico determinado por Goldstein y Brown, desde 1978, entre 25 y 50 mg/ dL.

Recientemente, las nuevas guías americanas para el manejo del colesterol han considerado varias controversias ${ }^{(28)}$.

\section{CONCLUSIONES}

Se debe establecer el mejor manejo y tratamiento, en forma integral, teniendo en cuenta, poblaciones especiales, condiciones de riesgo, daño en órgano blanco, cuadros especiales, enfermedades concominantes, para lograr tres enfoques fundamentales: prevenir eventos cardiovasculares, brindar protección de órganos blanco y mejorar la calidad de vida, mediante la detección temprana de la aterotrombosis subclínica. Es importante reconocer los factores de riesgo cardiovascular, estratificar y aplicar las escalas de medición de riesgo. La disminución del riesgo cardiovascular no solo es disminuir la presión arterial.

El tratamiento es global porque el riesgo es global. Es necesario no subestimar la aparición de menos de tres factores de riesgo del síndrome metabólico, fomentar el cambio en el estilo de vida en pacientes con presión arterial normal alta o prehipertensión. Los efectos deletéreos de la obesidad en el control de la presión arterial son asuntos de salud pública; el control y tratamiento de la dislipidemia es fundamental. En América Latina, la prevención cardiovascular no es una opción; es una obligación.

\section{REFERENCIAS BIBLIOGRÁFICAS}

1. Bryce A. Actualización y raciocinio del tratamiento antihipertensivo. An Fac med. 2010;71(4):251-5.

2. Framingham Heart Study. [Online].; 1950-2013 [citada 2014 Mayo]. Disponible en: http://www. framinghamheartstudy.org/about-fhs/aboutspanish.php.

3. Ruiz E. Riesgo y Prevención Cardiovascular. 1ra Edicion. Lima; 2014.

4. Naghavi M, Falk E, Hecht HS, Shah PK; SHAPE Task Force. The first SHAPE (Screening for Heart Attack Prevention and Education) guideline. Crit Path Cardiol. 2006 Dec;5(4):187-90.

5. Egan BM, Zhao Y, Axon RN. US trends in prevalence, awareness, treatment, and control of hypertension, 1988-2008. JAMA. 2010 May 26;303(20):2043-50. doi: 10.1001/jama.2010.650.

6. Sachdeva A, Cannon CP, Deedwania PC, Labresh KA, Smith SC Jr, Dai D, Hernandez A, Fonarow GC. Lipid levels in patients hospitalized with coronary artery disease: an analysis of 136,905 hospitalizations in Get With The Guidelines. Am Heart J. 2009 Jan;157(1):111-117.e2. doi: 10.1016/j. ahj.2008.08.010.

7. Loke KE, Curran CM, Messina EJ, Laycock SK, Shesely EG, Carretero OA, Hintze TH. Role of nitric oxide in the control of cardiac oxygen consumption in $\mathrm{B}(2)$-kinin receptor knockout mice. Hypertension. 1999;34(4 Pt 1):563-7.

8. Volpe M. Managing hypertension in cardiology practice according to risk profile. Int J Clin Prac. 2006;62:1403-12.

9. Kylin E. Studien uber das hypertonie-hyperglykamie-hyperurikamiesyndrom. Zentrablfinnere Med Leipz. 1923;81:105-27.

10. Expert Panel on Detection, Evaluation, and Treatment of High Blood Cholesterol in Adults. Third Report of the Expert Panel on Detection, Evaluation, and Treatment of High Blood Cholesterol in Adults. JAMA. 2001 Mayo 16;285(19):2486-97.

11. Grundy SM, Cleeman JI, Daniels SR, Donato KA, Eckel RH, Franklin BA, et al. Diagnosis and management of the metabolic syndrome. An American Heart Association/National Heart, Lung, and Blood Institute Scientific Statement. Executive Summary. Circulation. 2005;112:e285-90.

12. Grundy SM. Pre-diabetes, metabolic syndrome, and cardiovascular risk. J Am Coll Cardiol. 2012 Feb 14;59(7):635-43. doi: 10.1016/j. jacc. 2011.08.080

13. Deng Y, Scherer PE. Adipokines as novel biomarkers and regulators of the metabolic syndrome. Ann N Y Acad Sci. 2010 Nov;1212:E1-19. doi: 10.1111/j.1749-6632.2010.05875.x.

14. Schramm A, Matusik P, Osmenda G, Guzik TJ. Targeting NADPH oxidases in vascular pharmacology. Vascul Pharmacol. 2012 May-Jun;56(5-6):216-31. doi: 10.1016/j.vph.2012.02.012

15. López-Jaramillo $P$, Sánchez RA, Díaz M, Cobos L, Bryce A, Parra-Carrillo JZ, Lizcano F, Lanas F, Sinay I, Sierra ID1, Peñaherrera E, Benderky M, Schmid H, Botero R, Urina M, Lara J, Foos MC, Márquez G, Harrap S, Ramirez A. Latin american consensus on hypertension in patients with diabetes type 2 and metabolic syndrome. Clin Investig Arterioscler. 2014 Mar-Apr;26(2):85-103. doi: 10.1016/j.arteri.2013.11.008.

16. $2013 \mathrm{ESH} / \mathrm{ESC}$ Guidelines for the management of arterial hypertension. Rev Esp Cardiol. 2013 Nov; 66(11): p. 880.

17. James PA,Oparil S, Carter BL, Cushman WC, Dennison-Himmelfarb C, Handler J, et al. 2014 Eviden- 
ce-Based Guideline for the Management of High Blood Pressure in Adults Report From the Panel Members Appointed to the Eighth Joint National Committee (JNC 8). JAMA. 2014 Feb 5; 311(5): p. 507-20. doi: 10.1001/jama.2013.284427.

18. Assadi F. Prehypertension: a warning sign of future cardiovascular risk. Int J Prev Med. 2014 Mar;5(Suppl 1):S4-9.

19. Roberts WC, Bakris GL, Black HR, Sica DA, Sulkes DJ. The editor's roundtable: prehypertension. Am J Cardiol. 2009 Oct 15;104(8):1105-15. doi: 10.1016/j.amjcard.2009.07.010.

20. Huang Y, Cai X, Li Y, Su L, Mai W, Wang S, Hu Y, Wu Y, Xu D. Prehypertension and the risk of stroke: a meta-analysis. Neurology. 2014 Apr:82(13):115361. doi: 10.1212 WNL. 0000000000000268 .

21. Bryce A, Coca A. Controversia. Tratamiento de la hipertensión arterial: monoterapia o combinaciones. 2011 Julio-Agosto;79(4):355-63.

22. Bangalore S. Messerli FH, Wun CC, Zuckerman AL, DeMicco D, Kostis JB, LaRosa JC; Treating to New Targets Steering Committee and Investigators. J-curve revisited: An analysis of blood pressure and cardiovascular events in the Treating to New Targets (TNT) Trial. Eur Heart J. 2010 Dec;31(23):2897-908. doi: 10.1093/eurheartj/ ehq328.

23. Redon J, Mancia G, Sleight P, Schumacher H, Gao P, Pogue J, Fagard R, Verdecchia P, Weber M, Böhm M, Williams B, Yusoff K, Teo K, Yusuf S; ONTARGET Investigators. Safety and efficacy of low blood pressures among patients with diabetes: subgroup analyses from the ONTARGET (ONgoing Telmisartan Alone and in combination with Ramipril Global Endpoint Trial). J Am Coll Cardiol. 2012 Jan 3;59(1):74-83. doi: 10.1016/j.jacc.2011.09.040.

24. Zanchetti A. Evidence and wisdom: recom mendations for forthcoming guideline. J Hypertens. 2011 Jan;29(1):1-3. doi: 10.1097/ HJH.0b013e328341fb35.

25. Henry R. Black, MD, Raymond R. Townsend, MD, Michael A. Weber, MD. TheHeart.org. [Online].; 2014. Disponible en: http://www.medscape.com/ viewarticle/820407.

26. Hampton J. Evidence-based medicine, opinionbased medicine, and real-world medicin. Perspect
Biol Med. 2002;45(4):549-68

27. Tousoulis D, Psarros C, Demosthenous M, Patel R, Antoniades C, Stefanadis C. Innate and adaptive inflammation as a therapeutic target in vascular disease: the emerging role of statins. J Am Coll Csardiol. 2014 Jun 17;63(23):2491-502. doi: 10.1016/j.jacc.2014.01.054

28. Smith SC Jr, Grundy SM. 2013 ACC/AHA Guideline recommends fixed-dose strategies instead of targeted goals to lower blood cholesterol. J Am Coll Cardiol. 2014 Aug 12;12(64):601-12. doi: 10.1016/j. jacc.2014.06.1159.

\section{Conflictos de interés:}

El Dr. Juan Urquiaga Calderón es Medical Advisor de Laboratorios Sanofi-Perú.

Correspondencia:

Dr. Alfonso Bryce Moncloa

Correo electrónico: cardiogolf1@yahoo.es 